\title{
FACTORES CLAVE PARA LA BÚSQUEDA DEL APRENDIZAJE ORGANIZACIONAL EN LA CADENA DE SUMINISTRO: UNA APROXIMACIÓN TEÓRICA
}

\section{KEY FACTORS IN SUPPLY CHAIN'S ORGANIZATIONAL LEARNING: A THEORETICAL APPROACH}

Castillo Mateo, Belén (Universidad de Granada)

Tamayo Torres, Javier (Universidad de Granada) ${ }^{* *}$

Cabeza Pullés, Dainelis (Universidad de Granada) ${ }^{* * *}$

Roldán Bravo, María Isabel (Universidad de Jaén) ${ }^{* * *}$

Ruiz Moreno, Antonia (Universidad de Granada)

\section{RESUMEN}

Dada la importancia que ha tenido la subcontratación en los últimos años y el auge de las cadenas de suministro, gestionarlas de forma eficiente es un factor clave a tener en cuenta para mejorar el rendimiento de la empresa. Una buena relación con los clientes/proveedores aumenta el flujo de comunicación e información entre ambos, lo que les permite aprender unos de otros, logrando que haya aprendizaje organizacional y llegar a la obtención de una ventaja cooperativa que sea beneficiosa para ambos. Sin embargo, a pesar de ser muchas las ventajas de esta relación, las empresas son reacias a compartir información de su organización, encontrándose con la controversia de perder control y protección de su organización. El objetivo de este trabajo es construir un marco teórico que permita explicar las variables que determinan el grado de influencia del aprendizaje organizacional dentro de la cadena de suministro. La metodología seguida ha sido una revisión de la literatura previa que nos ha servido de apoyo para cumplir el objetivo propuesto. Como resultado de este trabajo, hemos detectado que los factores clave para la búsqueda del aprendizaje organizacional en la cadena de suministro se agrupan en tres: externos a la organización, internos a la misma y relacionales, inherentes a la relación que mantiene con los integrantes de la cadena de suministro a la que pertenece.

Palabras claves: Cadena de suministro, aprendizaje organizacional, ventaja competitiva, empresa, subcontratación. JEL: M11.

\section{ABSTRACT}

Given the significant role played by subcontracting in recent years and the rise of supply

\footnotetext{
* Departamento de Organización de Empresas, Facultad de Ciencias Económicas y Empresariales, Cartuja s/n, 18071, Granada.

* Departamento de Organización de Empresas, Facultad de Ciencias Económicas y Empresariales, Cartuja s/n, 18071, Granada. jatamayo@ugr.es

*** Departamento de Organización de Empresas, Facultad de Ciencias Económicas y Empresariales, Cartuja s/n, 18071, Granada. dainelis@ugr.es

**** Departamento de Economía Financiera y Contabilidad. Universidad de Jaén, Campus Las Lagunillas, s/n, 23071, Jaén. mrbravo@ujaen.es

***** Departamento de Organización de Empresas, Facultad de Ciencias Económicas y Empresariales, Cartuja s/n, 18071 Granada. aruizmor@ugr.es

Recibido: Abril de 2016. Aceptado: Mayo de 2016.
} 
chains, manage them efficiently is a key factor to consider for improving business performance. A good relationship with customers / suppliers increases the flow of communication and information between them, allowing them to learn from each other, making and organizational learning have come to obtain a cooperative advantage that is beneficial to both. However, despite being many advantages of this relationship, companies are reluctant to share information about your organization, meeting the controversy of losing control and protection of your organization. The objectives of this work are to analyze the influence of organizational learning within the supply chain because it improves the situation of the company, not only in terms of reducing costs but also optimize performance, consider some of the variables that influence this learning and suggest several proposals, laying the groundwork for future research.

Keywords: Supply chain, organizational learning, competitive advantage, business, outsourcing. JEL: M11.

\section{INTRODUCCIÓN}

La idea de que el éxito de una organización depende en mayor medida de la capacidad de la cadena de suministro a la que pertenece más que a sus propias capacidades está ganando mucha aceptación (Chow et al., 2008; Green et al., 2006). La posición competitiva y la calidad de la cadena de suministro se ven reforzados por la competencia que cada organización tiene para gestionar la cadena. Esta competencia se construye a través de cómo se adoptan las prácticas y se resuelven los problemas en la gestión de la cadena de suministro (Chow et al., 2008). Todo ello es el resultado de un aprendizaje continuo para la organización (Spekman et al., 2002), que permite a la organización atender la demanda, es decir, ser más flexible y responder a las cambiantes demandas del mercado que atiende, así como lograr la excelencia en la cadena de suministro en su zona de operaciones (Kuei et al., 2005).

La ineficiencia de la cadena de suministro ha sido identificada como uno de los problemas más frecuentes a los que se enfrentan las organizaciones (Lewis, 2005). Un ejemplo donde se refleja el valor que tiene para una empresa su cadena de suministro lo encontramos en el estudio de Hendricks y Singhal (2003) donde, tras anunciar un importante problema en la cadena de suministro, se erosiona el valor de mercado de la empresa en un promedio del 10 por 100 , con lo cual, para aumentar el valor y el rendimiento de ésta, es imprescindible gestionar adecuadamente la cadena de suministro.

Además, en los últimos años el mercado global se ha vuelto muy volátil, los clientes exigen precios más bajos, una entrega más rápida, de mayor calidad y aumentar la variedad de productos (Narasimhan y Das, 1999; Vokurka y Lummus, 2000). Otra característica del mercado actual es que se caracteriza por altos niveles de turbulencia e incertidumbre. Las organizaciones se enfrentan a crecientes presiones de la competencia con respecto a los precios, entrega, calidad, variedad e innovación de productos y servicios. Con el fin de responder a estos retos, las organizaciones están buscando la agilidad de una manera sistemática en sus cadenas de suministro.

Es generalmente conocido que en un entorno turbulento y dinámico las empresas tienen que desarrollar capacidades que les permita ser más ágiles y flexibles y, al mismo tiempo, puedan incorporar nuevos productos y procesos y nuevas tecnologías que les permitan desarrollar y explotar mejores prácticas en las cadenas de suministro. Esta agilidad y flexibilidad llama a las empresas a aumentar su efectividad, explotar sinergias y aprender a lo 
largo de todas las áreas de sus operaciones (Hyland et al., 2003). Uno de los objetivos por lo que el aprendizaje organizacional y la transferencia de conocimiento adquieren suma importancia es porque la cadena de suministro puede lograr esa efectividad y flexibilidad, obteniendo mejores resultados siempre y cuando el desarrollo de canales efectivos, la visión de los miembros, las estrategias y las operaciones sean en la misma dirección (Hult et al., 2004). Para que la visión de los miembros sea en la misma dirección deben tener una visión compartida, que se refiere a la idea que todos los miembros de una organización tengan la misma visión y un enfoque común que les permita desarrollar respuestas a las tendencias del mercado y eventos inciertos (Sinkula et al., 1997).

El modelo de aprendizaje organizacional tiene su raíz en la escuela principal de pensamiento basado en la teoría de recursos y capacidades de la empresa. El punto de vista basado en los recursos de la empresa (Penrose, 1959; Wernerfelt, 1984) sostiene que las principales ventajas competitivas de las empresas residen en sus capacidades, sus procesos y otros recursos intangibles (como la cultura corporativa, marcas y reputación). Bajo este punto de vista, entendemos el aprendizaje organizacional y la gestión del conocimiento como funciones de la organización que les permite compartir y utilizar conocimiento para obtener ventajas competitivas (Dong y Yang, 2015). Dada la importancia de la búsqueda del aprendizaje organizacional en la gestión de la cadena de suministro, este trabajo tiene como objetivo principal conocer las variables que explican la misma, haciendo especial hincapié en el intercambio de información en el contexto de la cadena de suministro. Se pretende pues, responder, a la siguiente pregunta de investigación: ¿Qué variables influyen en la búsqueda del aprendizaje organizacional en la cadena de suministro?. Para ello, la metodología seguida ha sido la revisión de la literatura más reciente y relevante en el ámbito de nuestro estudio.

La estructura que tiene este trabajo es la siguiente: tras un primer apartado en el que se revisa la literatura sobre el aprendizaje organizacional en la cadena de suministro, se presenta una propuesta de marco teórico en el que se exponen los factores clave para la búsqueda del aprendizaje organizacional en la cadena de suministro. Por último, se presentan las conclusiones de este trabajo y se proponen futuras líneas de investigación.

\section{REVISIÓN DE LA LITERATURA: EL APRENDIZAJE ORGANIZACIONAL EN LA CADENA DE SUMINISTRO}

Como hemos avanzado, la propuesta de modelo teórico que constituye el objetivo de nuestro trabajo parte de una revisión de la literatura previa para conocer el estado de la cuestión al respecto. Para ello, hemos analizado la literatura más reciente y relevante del ámbito de nuestro estudio: aprendizaje organizacional en cadena de suministro. Se han revisado artículos científicos indexados por JCR Science Edition y JCR Social Sciences Edition en el período comprendido entre 1973-2015. En una primera fase, se analizó la importancia de compartir información en la cadena de suministro y los problemas para ello. A continuación se centró la atención en el concepto de aprendizaje organizacional y cadena de suministro para, finalmente, relacionar los factores que promueven la transferencia de conocimiento y, en consecuencia, el aprendizaje organizacional en el contexto de una cadena de suministro. Hemos agrupado dichos factores en tres: factores externos, factores relacionales y factores internos y se exponen en el apartado Marco Teórico de este trabajo. 


\subsection{Importancia de compartir información en la cadena de suministro y problemas para ello. El intercambio de información como promotor del aprendizaje organizacional}

Las empresas se centran cada vez más en lo que hacen bien, en sus actividades principales, y subcontratan aquellas actividades secundarias o no esenciales a proveedores, por lo tanto, cada vez más dependen de sus socios en la cadena de suministro, un problema con éstos puede ocasionar un gran perjuicio para la empresa.

Esta dependencia de otras empresas hace que la importancia de compartir información y aprender mutuamente no sólo presente una oportunidad, sino también un desafío (Spekman et al., 2002), si cede información confidencial de su empresa susceptible de ser vulnerable frente a posibles comportamientos oportunistas.

La información es importante, pero convertir esa información en conocimiento suministra las bases para una gestión mejor (Hult et al., 2004), la gestión efectiva de la transferencia de conocimiento puede ser vista como una competencia esencial que potencialmente otorga una ventaja competitiva a los miembros de la cadena de suministro (Spekman et al., 2002), así un tema estratégico clave es la habilidad para aprovechar las capacidades de tu socio más allá de los activos tangibles y el conocimiento explícito. Hay un conjunto de activos o habilidades que permanecen tácitos y son menos fácilmente transferibles dentro de la cadena de suministro. Algunos de estos activos incluyen el saber hacer de los empleados, la reputación y la cultura que reside en la estructura de la empresa (Hall, 1999). Dainty y Brooke (2004) defienden la gestión de la cadena de suministro para poner de relieve oportunidades de negocio, ya que las organizaciones puedan capturar, cuestionar y transferir eficiencia de la cadena de suministro a otros proyectos. El intercambio de información puede ayudar a la familiarización y dar la oportunidad de desarrollar una mentalidad común de temas (Hult et al., 2004). Spekman et al. (2002) apuntaban que en las cadenas de suministro integradas y en todo tipo de alianzas, la habilidad para absorber y transferir conocimiento ofrece ventajas que superan el resultado de reducir costes únicamente, algunos de los beneficios clave son construir confianza (Kumaraswamy et al., 2008) y reducir los costes transaccionales.

Últimamente las grandes empresas están reescribiendo las reglas de la estrategia y de la rivalidad en sus industrias mediante el uso de las cadenas de suministro, no sólo como un mecanismo para obtener, transformar y distribuir materiales, sino también como un arma competitiva, para compartir beneficios, crecimiento, cuota de mercado y otros indicadores clave (Handfield y Nichols, 2002). Las empresas reconocen la importancia de mejorar el rendimiento de la cadena de suministro (Knowles et al, 2005), para ello la organización debe desarrollar e implementar una adecuada estrategia de gestión de la cadena de suministro.

Hult et al. (2007) señalan que una de las tendencias centrales hoy en día en la economía es que la competencia es menos empresa versus empresa y cada vez más cadena de suministro versus cadena de suministro. Hoy en día las empresas toman conciencia de que no pueden competir efectivamente de forma aislada de sus proveedores y de otras entidades en la cadena de suministro (Hult et al., 2007; Hernández et al., 2010). Un ejemplo de esto son las empresas de alta tecnología, que encuentran en la transferencia de conocimiento una virtud esencial debido a su ritmo rápido y su entorno de gran incertidumbre (Hagedoorn, 1993). Los cambios en la tecnología son un importante impulsor de cambios estratégicos y estructurales. Grantham et al. (1997) advierten de la capacidad de difusión de la tecnología para generar temas importantes como el aumento del flujo de información y el aumento del ritmo en la actividad. 
Uno de los problemas por los que no se ha prestado demasiada atención hasta ahora a la gestión de la cadena de suministro y al aprendizaje organizacional es que muchos directivos equiparaban únicamente la gestión de la cadena de suministro con la reducción de costes (Maloni y Benton, 1997). Sin embargo, la gestión de la cadena de suministro es una estrategia que nos ayuda a mejorar su rendimiento, además, es fundamental para crear mayor valor para el cliente si se sabe cómo hacer propuestas competitivas y atractivas de valor no sólo para clientes y proveedores de la empresa, sino también para los clientes de esos clientes y los proveedores de esos proveedores (Lusch, 2011).

La idea de subcontratar aumenta la discusión que existe entre la dependencia de los proveedores por su experiencia y la preocupación por su comportamiento oportunista (Quinn, 1999). Las empresas flaquean en sus relaciones por tener miedo a la pérdida de control y porque no reconocen las ganancias atribuibles a asociaciones eficaces en la cadena de suministro. Por ejemplo, Maloni y Benton (1997) analizan en las relaciones internacionales de las empresas americanas; cada socio se esfuerza por aprender del otro mientras simultáneamente está protegiendo su conjunto de competencias internas. Esta tensión tiene impacto negativo en el aprendizaje y en el logro de una ventaja cooperativa (Lei et al., 1997).

Entender cómo se puede aprender más rápido y luego actuar en ese aprendizaje es clave para tener continuamente una ventaja competitiva sobre el conocimiento (Lusch, 2011; Dong y Yang, 2015). En cambio, las empresas protegen su información celosamente y la mayoría de la información la comparten a regañadientes. Las empresas no suelen permitir a sus socios de la cadena de suministro estar demasiado cerca de ellos por el miedo a que expropien sus secretos (Spekman et al., 2002), un estudio de Ernst \& Young del rendimiento de las cadenas de valor refleja que un tercio de los encuestados sostuvo que no compartían información estratégica, ya sea con sus proveedores o clientes, y el 39 por 100 no tenían información de la orden de venta de acciones o información de logística de distribución con sus proveedores (O’Laughlin, 2000). Una de las principales preocupaciones de la relación del aprendizaje común es que la divulgación de conocimientos puede plantear la amenaza de explotar el conocimiento sobre la propiedad de una manera no deseable (Dyer y Nobeoka, 2000), es decir, de manera oportunista.

Spekman et al. (2002) también apoyan la idea de que existen tensiones entre la cooperación/integración de la cadena de suministro y el miedo al oportunismo. Otro de los inconvenientes es que es particularmente difícil para los pequeños proveedores desarrollar mecanismos de gobierno eficaces y elaborar estrategias que salvaguarden su relación con grandes clientes (Subramani y Venkatraman, 2003). Además, las distancias culturales y geográficas entre socios atenúan la eficacia de herramientas de gobierno legal, como contratos formales (Cavusgil et al., 2004), por lo que la confianza, el compromiso y la comunicación ayudan a controlar estos temores y a mejorar el rendimiento (Spekman et al., 2002). Según Lusch (2011), se podría desarrollar un sistema de comunicación global que permitiese a las distintas culturas operar en todo el mundo para trabajar como un equipo eficaz y proteger los derechos de propiedad importantes.

Una vez que se ha comprendido la importancia del aprendizaje organizacional en la cadena de suministro nos parece acertado analizar en más profundidad los conceptos que queremos estudiar y sus definiciones (aprendizaje organizacional, cadena de suministro y la capacidad que permite que ocurra) para afianzar estos conocimientos y buscar qué factores son necesarios para que se dé el aprendizaje organizacional. 


\subsection{Aprendizaje organizacional}

Podemos encontrar distintas definiciones de aprendizaje organizacional teniendo en cuenta la literatura previa sobre cadenas de suministro, ya que el aprendizaje organizacional ha sido testigo de la creciente aceptación dentro de la comunidad empresarial (Styhre et al., 2004). Alguna definición de las más reconocidas y aceptadas por lo académicos es la de Levitt y March (1988), que entendían el aprendizaje organizacional como una rutina basada en la historia y orientada hacia objetivos. El aprendizaje organizacional también se define como el desarrollo de nuevos conocimientos o comprensión que potencialmente tiene influencia en el comportamiento de la empresa, o como la adquisición, interpretación y difusión de información de la organización dentro de la cultura de la empresa (Slater y Narver, 1995). Para que se dé aprendizaje organizacional es clave la capacidad de absorción, entendida como la acumulación de conocimiento relacionado que permite la evaluación y explotación de los desarrollos posteriores (Cohen y Levinthal, 1990). La capacidad de absorción de información se entiende como una capacidad dinámica que permite a las empresas entender la tecnología del conocimiento y predecir su futura aplicación (Zahra y George, 2002), dicha capacidad aumenta la innovación (Lane y Koka, 2006) y conduce a mejores resultados empresariales (Tsai, 2001).

A su vez el proceso de aprendizaje organizacional puede ser visto como una interacción dinámica entre las creencias, conductas y factores ambientales (Crossan y Inkpen, 1995). Lennon y Wolin (2001) sostuvieron que el aprendizaje organizacional es más difícil de duplicar o imitar. Teniendo en cuenta este último concepto de aprendizaje organizacional, nos encontramos que en muchos estudios se refleja el aprendizaje organizacional como una fuente de competitividad (Martínez-Costa y Jiménez-Jiménez, 2009). La relación efectiva de intercambio de aprendizaje entre socios puede crear capacidades dinámicas de competitividad sostenible para las empresas en ambientes que cambian rápidamente, Eisenhardt y Martin (2000) sugieren que una capacidad de aprendizaje de la empresa también puede ser vista como una capacidad dinámica (Teece et al., 1997).

Es por ello que cada vez más, se hace hincapié en arraigar los mecanismos de aprendizaje organizacional en las cadenas de suministro ya existentes (Stuart y Fernie, 2013). El motivo de que el aprendizaje organizacional pueda ser visto como una capacidad dinámica que puede crear dentro de la empresa una ventaja competitiva, es porque las empresas aprenden de forma distinta, las competencias relacionadas con el aprendizaje no se distribuyen de forma homogénea ya que unas empresas son mejores desarrollando capacidades y/o son más expertas en el acceso y la capacidad de interiorización que tienen otras (Hamel, 1991). Cada organización tiene diferencias en su forma de interpretar la información (Daft y Weick, 1984), en su forma de recordarlo (Easterby-Smith, 1997) y en su forma de usar ese aprendizaje (Brown y Duguid, 2001). Estas diferencias en el aprendizaje también se dan por factores contextuales tales como el liderazgo (Vera y Crossan, 2004), la cultura (Popper y Lipshitz, 2000), la estrategia, la estructura y el entorno (Fiol y Lyles, 1985). El aprendizaje organizacional puede servir como una estrategia de equilibrio que ayuda a proteger y reestructurar la relación con el cliente grande y crear valor de la relación (Subramani y Venkatraman, 2003), el aprendizaje ayuda a crear valor en la relación gracias a la retroalimentación que debe de darse entre las empresas, según Crossan et al. (1999) los individuos, los grupos y las organizaciones aprenden a través de la retroalimentación, diferentes procesos de intuición, interpretación, integración e institucionalización. 
Según Hult et al. (1998), existen cuatro dimensiones del aprendizaje organizacional tales como la orientación del equipo, los sistemas de orientación, la orientación al aprendizaje y la memoria de la cadena de suministro. Por otro lado, Myers y Cheung (2008) encuentran tres tipos de aprendizaje organizacional dentro de la cadena de suministro global, cada uno con distintos beneficios: intercambio de información, sentido conjunto de decisiones e integración del conocimiento. Simonin (2004) propone características organizativas, como la intención de una empresa de aprendizaje, la capacidad de aprendizaje y la ambigüedad del conocimiento, como factores clave para que se dé aprendizaje organizacional dentro de la empresa. Una organización debe tener una orientación hacia el aprendizaje, no sólo reunir y difundir información sobre los mercados, sino también examinar constantemente la calidad de sus funciones de almacenamiento y de interpretación (Baker y Sinkula, 1999).

Es importante especificar qué entendemos por conocimiento ya que el aprendizaje organizacional se basa en la transferencia de conocimiento; en este sentido atendemos a varias descripciones del conocimiento, como la información que se selecciona y combina con experiencia y opinión de expertos para rendir una opinión o una interpretación de una situación que podría no ser clara para todos (Spekman et al., 2002). Conocimiento se refiere a la información que se enriquece a través de la interpretación, el análisis y el contexto en el que se examina (Hyland et al., 2003). La interpretación de la información, o sentido común, es el proceso por el cual los miembros desarrollan un entendimiento común acerca de datos y eventos (Corner et al., 1994), entendiendo por información los datos que se han organizado, analizado e interpretado, ya sea por ordenadores o por personas (Hyland et al., 2003). Desde la perspectiva de recursos y capacidades, se entiende el conocimiento como una capacidad única, capaz de crear y explotar la sabiduría mejorando los resultados (Grant, 1996); siguiendo esta definición el conocimiento es el mayor activo estratégico y no imitable de la empresa y los activos de conocimiento son el factor clave de la competitividad continua (Grant, 1996).

Desde una visión más práctica, distinguimos dos tipos de conocimiento dentro de la empresa, conocimiento tácito o explícito. El conocimiento tácito es aquel que tienen las personas interiorizado en su modelo mental, creencias, costumbres, experiencias, por lo tanto, es difícil de trasmitir. En cambio, el conocimiento explícito es fácilmente comunicado y distribuido, este conocimiento se mantiene formalmente en los procedimientos de las políticas, sistemas y registros históricos y financieros (Lee y Yang, 2000). Esta distinción de conocimiento es congruente con los diferentes niveles de la organización, ya que los conocimientos se acumulan de forma distinta (Vera y Crossan, 2004) para cada trabajador que para el conjunto de la organización.

A nivel individual, el conocimiento es incrustado en los individuos como competencias, capacidades y motivación, a nivel de grupo, el conocimiento es representado en la comunicación, el lenguaje, las historias y la comprensión compartida y, al nivel organizativo, el conocimiento se basa en los sistemas, estructuras, procesos, rutinas, estrategias, cultura y patentes de la organización (Crossan et al., 1999; Vera y Crossan, 2004). Como el conocimiento de una empresa comprende estas acciones de conocimiento, su acumulación aumenta el conocimiento total de la empresa. Algunas veces cuando el conocimiento es tácito y no es fácilmente transferible o es imposible de imitar, la única esperanza de tener realmente acceso a esos conocimientos es aprender del comportamiento del socio de la cadena de suministro, dado que si no se puede transferir oralmente sólo se puede aprender a través de la experiencia (Spekman et al., 2002).

Ya que nosotros atendemos al conocimiento dentro de la cadena de suministro es adecuado referirnos al capital intelectual, que se define como el conocimiento y la capacidad 
de saber de una colectividad social, como una organización, comunidad intelectual, o de la práctica profesional (Laine y Laine, 2012). Las competencias esenciales de una empresa radican en la acumulación de capital intelectual, en resumen, su tecnología, experiencia, habilidades y gestión de procesos (Hamel, 1991). El capital intelectual consiste en tres componentes (Subramaniam y Youndt, 2005):

1. Capital humano: conocimiento, destrezas y habilidades individuales

2. Capital organizacional: bases de datos, estructuras, rutinas, sistemas y procesos

3. Capital social: estructura de relaciones entre los actores

Hoy en día el capital intelectual se ha convertido en uno de los principales factores que agregan valor a la empresa. La capacidad de ésta para activarlo depende en gran medida de la cantidad de experiencias de aprendizaje anteriores con el mismo tipo de conocimiento (Cohen y Levinthal, 1990), debido a que el capital intelectual surge de la interacción entre los conocimientos que posee la empresa y va más allá de su simple acumulación.

Un factor importante para que se active el capital intelectual es la distribución de la información, para que haya conocimiento es indispensable que se distribuya la información; se entiende por distribución de información el proceso por el cual se comparte información de diferentes fuentes. En las cadenas de suministro este intercambio se produce en toda la cadena, incluyendo sus diferentes nodos y miembros (Kohli et al., 1993) abarcando así a distintos actores. El desarrollo de conocimiento es un fenómeno en el que los actores conducen a la adquisición de conocimientos, distribución de información, sentido común y memorización de dicho conocimiento en la cadena de suministro (Hult et al., 2004; Huber, 1991).

Para tal memorización, es importante tener en cuenta la dimensión de memoria de la cadena de suministro, dicha memoria se define como la cantidad de conocimiento, experiencia y familiaridad con el proceso de la cadena de suministro, sus operaciones y comportamientos; sirve como mecanismo por el cual el conocimiento se almacena para uso futuro estratégico y, como tal, es fundamental como punto de partida para comportamientos de aprendizaje futuros (Hult et al., 2007).

Para fomentar este comportamiento de aprendizaje es aconsejable un clima de comunicación positivo, que implica una cultura participativa, fomenta la toma de riesgos individuales (Roth et al., 1994), demuestra el compromiso de liderazgo para el desarrollo de habilidades (Levinson y Asahi, 1995) e implementa mecanismos que promuevan el diálogo externo (Goes y Park, 1997). Una orientación de aprendizaje permite a las empresas no solo aprender una nueva información, sino también, a través de una visión común, permite un enfoque sobre lo que aprender (Sinkula et al., 1997).

\subsection{Cadena de suministro}

Una clasificación básica de la gestión de la cadena de suministro revela dos escuelas con pensamientos muy distintas:

- Escuela funcional (Spekman et al., 1998), que mantiene una estrecha relación con los principios fundamentales de la gestión de la cadena de suministro, como son las compras, la logística y la gestión de las operaciones (Lummus y Vokurka, 1999), en esta escuela se trata de aprovechar el mejor precio posible en las condiciones actuales del mercado, a través de la ruta óptima de adquisición (Spekman et al., 1998)

- Escuela filosófica (Cooper y Ellram, 1993), que reafirma muchas de las características clave de la escuela funcional, además articula las características tácitas tales como la 
integridad, trabajo en equipo y profesionalidad. Para esta escuela la principal ambición de la gestión de la cadena de suministro es mejorar el rendimiento, independientemente de la competencia (Power, 2005).

Tradicionalmente una cadena de suministro era vista como una red de actores que transforman materia prima en productos distribuidos (Bowersox et al., 1999), pero en los últimos años las cadenas de suministro se han visto como mecanismos tácticos de coordinación de logística, de flujos de trabajo y de información relacionada; la gestión de la cadena de suministro ha crecido hasta abarcar una realidad competitiva (Handfield y Nichols, 2002), en su gestión han destacado las áreas de compras (o adquisiciones), gestión de materiales, almacenaje y control de inventarios, distribución, transporte y logística de transporte (Hyland et al., 2003), ya que han sido sus principales funciones durante mucho tiempo, además la capacidad de fabricación se divide normalmente en cinco dimensiones: coste, calidad, desarrollo de productos, entrega y flexibilidad (Tukamuhabwa et al., 2011), por lo que habitualmente se atendía a estas cinco dimensiones para elegir un socio en la cadena de suministro.

Hoy en día encontramos nuevos factores, y aunque una cadena de suministro es vista como un sistema de procesamiento de productos, tal vez sea igualmente valioso visualizarla como un sistema de procesamiento de información e interpretación (Daft y Weick, 1984), ya que el procesamiento de la información en la gestión de la cadena de suministro es crítico para su éxito (Bowersox et al.,1999), las empresas ahora compiten como socios colaboradores que aportan valor, y juntos combinan sus habilidades, capacidades y experiencias para lograr los objetivos que no podrían lograr por ellos solos (Spekman et al., 2002). Esto conlleva un nuevo reto al que deben enfrentarse las empresas, el de gestión y transferencia de la información con sus socios (Hyland et al., 2003) a lo largo de toda la cadena de suministro, puesto que dentro de la misma podemos encontrar distinto niveles de proveedores. Park y Hartley (2002), indicaron que las prácticas de la cadena de suministro de proveedores de primer nivel ${ }^{1}$ afectan el desempeño de los proveedores de segundo nivel, de la misma forma, apoyaron la idea de que las mejores prácticas de la cadena de suministro deben propagarse a través de toda la cadena de suministro para mejorar su rendimiento global.

La gestión de la cadena de suministro se ve como una integración de procesos del negocio desde el usuario final hasta los proveedores originales de los productos, los servicios de información añaden valor a los clientes y a otras partes interesadas (The Logistic and Distribution Institute, 2000). La capacidad de gestión de la cadena de suministro es un medio para mejorar y mantener su competitividad (Udin et al., 2006). Roh (2008) apoya esta idea, ya que, según su estudio, la gestión de la cadena de suministro es importante para obtener resultados exitosos de ésta. Cada vez más, las cadenas de suministro requieren continua distribución de información con el fin de mantener la integración estratégica, operativa y tecnológica (Hult et al., 2004). Las cadenas de suministro que utilizan benchmarking para evaluar el rendimiento relativo tienen creencias fuertes de que necesitan conocimientos adicionales a través de sus sistemas (Bowersow et al., 1999). Hay que tener en cuenta que las cadenas de suministro carecen de muchos de los mecanismos establecidos, formales e

\footnotetext{
${ }^{1}$ Proveedores de primer nivel son aquellos con los que la empresa lleva a cabo un acuerdo de colaboración y tiene relación directa con ellos. En cambio, los de segundo nivel son el siguiente eslabón en la cadena de suministro, por decirlo así, son los proveedores de nuestros proveedores, con los que la empresa además no tiene un trato directo. De esta forma nos encontramos cadenas de suministro de primer nivel (con sólo un nivel de proveedores) u otras, de segundo o tercer nivel según aumenta la complejidad de la cadena de suministro por el incremento de sus miembros.
} 
informales, que guían las decisiones de las empresas, dada la falta de culturas profundamente arraigadas en las cadenas de suministro, el significado compartido se convierte en un mecanismo fundamental para garantizar la coordinación. Por ello, es importante la coordinación de conocimientos dentro de la cadena de suministro, dicha coordinación a través de los nodos reduce duplicaciones, pérdidas y redundancias (Hult et al., 2004).

Una cadena de suministro de aprendizaje puede ser definida como una cadena de suministro integrada que tiene una capacidad dinámica, la capacidad de tener agilidad añadida a aprender y responder de los cambios en el entorno, logrando así una cadena de suministro estratégica, una cadena de suministro estratégica se forma por un esfuerzo para crear una fuente rara, valiosa, e irrepetible de conocimiento y coordinación (Grant, 1996). Las estrategias de la cadena de suministro dependen de una estrecha interacción con la comercialización y las ventas de los recursos, procesos y habilidades en la empresa. La colaboración entre empresas en los procesos de abastecimiento da lugar a ahorro en costes, flexibilidad y reducción de tiempo de espera, estos aspectos conducen a la mejora de la cadena de suministro, su gestión y su rendimiento. Las construcciones de relaciones de colaboración son intercambios de información, sincronización de las decisiones y alineación de incentivos que dan lugar a una mayor colaboración.

Con el aumento de la subcontratación y el cambio hacia una cadena de suministro impulsada por la demanda, los proveedores se encargan de las actividades de valor añadido más alto y necesitan desarrollar capacidades dinámicas específicas para atender a sus clientes globales exigentes (Jean et al., 2010).

No podemos entender el aprendizaje organizacional dentro de la cadena de suministro si no atendemos al concepto explicado anteriormente de aprendizaje como una capacidad, y que además no podría darse sin otras capacidades que debe poseer la cadena de suministro. El factor clave para comprender la importancia del aprendizaje organizacional radica en el hecho de que las capacidades dinámicas proveen a las empresas de ventajas competitivas. Por lo tanto, nos parece adecuado hacer referencia al concepto de capacidad relacionado con el aprendizaje y con la cadena de suministro.

Las capacidades se describen como acciones integradas en los recursos acumulados a través del aprendizaje, se incluyen las conductas interiorizadas, las habilidades técnicas, las rutinas organizativas y los activos corporativos (Hyland et al., 2003). Las capacidades clave que son fundamentales para el aprendizaje y la mejora continua en los centros de distribución son, según Hyland et al. (2003):

- la gestión del conocimiento

- la gestión de la información

- la capacidad de alojar y gestionar la tecnología y los problemas asociados

- la capacidad de gestionar las operaciones de colaboración

Para mantener una ventaja competitiva sostenible en estas funciones de logística, como en los centros de distribución, la gestión de las operaciones deben introducir una mejora continua y la garantía de que sus organizaciones tienen las capacidades necesarias para fomentar el aprendizaje organizacional (Hyland et al., 2003)

Una vez vistos estos conceptos pasamos a la búsqueda de variables que influyen en el aprendizaje organizacional dentro de la cadena de suministro. 


\section{MARCO TEÓRICO}

\subsection{Factores externos que explican el aprendizaje organizacional en la cadena de suministro}

La literatura previa integra un número de variables que contribuyen a la transferencia de conocimiento en la cadena de suministro y que producen menos conflicto y oportunismo, mayor cooperación y un aumento del desempeño de los miembros de la cadena (Goffin et al., 1997; Brown y Hendry, 1997). Es conocido que las características que dan lugar a una mayor colaboración entre los socios de la cadena de suministro conducen a un mayor aprendizaje y apoyan las estructuras y procesos que promueven y apoyan el aprendizaje (Spekman et al., 2002). Por lo que el aprendizaje se ve afectado por la capacidad de absorción del socio (Cohen y Levinthal, 1990), la capacidad de absorción se ve afectada por conocimientos relacionados con anterioridad, la red de comunicación, el clima de comunicación y los mecanismos de exploración (Tu et al., 2006). Por otro lado, una mayor absorción aumenta la probabilidad de la integración de la innovación externa por el fabricante y permite efectos indirectos de conocimiento secundario (Harhoff, 1996; Dyer y Nobeoka, 2000).

Las rutinas de las organizaciones (Nelson y Winter, 1982), sus culturas internas (Schein, 1984) e, incluso, las instituciones (Zysman, 1994) en las que confían, se crean gradualmente con el tiempo. Por todo ello, a pesar de que el concepto de colaboración en la cadena de suministro es nuevo para la mayoría de sus miembros, los socios siguen tratando de averiguar cómo funcionan estas nuevas relaciones y cuáles son las expectativas. Se conoce que, las características de confianza y compromiso en la relación son un ingrediente esencial en cualquier relación de colaboración a largo plazo (Spekman et al., 2002). Sin embargo, la investigación ha identificado activos relacionales, como la confianza y el aprendizaje organizacional, como temas clave en la gestión eficaz de las relaciones internacionales del canal de suministro (Griffith et al., 2008; Wu et al., 2007). Así, Selnes y Sallis (2003) destacan tres antecedentes: la incertidumbre del entorno, la confianza y el compromiso, como determinantes de la relación de aprendizaje.

\subsubsection{Incertidumbre del entorno}

La incertidumbre del entorno es el grado de imprevisibilidad de los cambios que se dan en el ambiente externo de la organización y que afectan a la empresa. Esta incertidumbre viene dada por el grado de complejidad, turbulencia, diversidad y hostilidad del entorno, entendiendo por entorno todo aquello que está en permanente contacto con la empresa al ser un sistema abierto.

Lee et al. (2008) y Selnes y Sallis (2003) vincularon la incertidumbre, incluyendo la turbulencia del entorno y la intensidad de la competencia, al desarrollo de las actividades de aprendizaje organizacional. Teniendo en cuenta esta turbulencia, (Levinthal y March, 1981) analizaron que cuanta mayor es la turbulencia que hay en los mercados aumentan los requisitos para que se desarrolle el conocimiento en la cadena de suministro. Por otro lado, Hult et al. (2007) analizaron en su estudio la turbulencia como un efecto moderador, donde destacaron que la misma influye positivamente en la relación rendimiento-desarrollo de conocimientos y negativamente en la correspondiente a rendimiento-cultura competitiva.

Las relaciones a largo plazo entre los miembros de la cadena de suministro se han desarrollado para proporcionar estabilidad, pero esos vínculos se rompen cuando se necesita un cambio, aunque se busca previsibilidad, no a costa de la creación de inflexibilidad (Hult et 
al., 2004). Las empresas están dispuestas a compartir conocimiento más importante para superar problemas de adaptación derivados de la incertidumbre del entorno, incluidas las dificultades en los volúmenes de ventas de previsión y la volatilidad de las ventas y cuota de mercado (Selnes y Sallis, 2003). Es por ello, que planteamos que:

P1: En situaciones de incertidumbre del entorno las empresas comparten más conocimiento entre ellas, favoreciendo así el aprendizaje organizacional en la cadena de suministro.

\subsection{Factores relacionales que explican el aprendizaje organizacional en la cadena de suministro}

\subsubsection{Confianza}

Según (Selnes y Sallis, 2003) otro de los factores clave para que se dé transferencia de información en la cadena de suministro es la confianza, entendiendo por confianza la creencia en que el otro miembro actuará de una manera predecible, mantendrá su palabra y se comportará de una forma que no afectará al otro (Spekman et al., 2002). En este sentido, la confianza permite a un miembro ponerse en riesgo a sabiendas que su pareja no actuará en su propio interés (Gulati, 1995). La confianza produce que compradores y vendedores trabajen conjuntamente y este sentido de compromiso lleva a dicha relación a un nivel en el que se pueden combinar los recursos para lograr el beneficio mutuo (Spekman et al., 2002).

La confianza juega un papel crucial en el aprendizaje facilitando la relación entre empresas, ya que los potenciales comportamientos oportunistas y las tensiones creadas por el intercambio de conocimientos confidenciales se pueden reducir mediante un alto nivel de confianza entre los socios del intercambio (Wu et al., 2007). De acuerdo con la teoría del intercambio relacional, un importante nivel de confianza puede ser visto como un mecanismo de gobierno organizacional que ayuda a disuadir comportamientos oportunistas, porque la confianza ayuda a disminuir la vulnerabilidad percibida entre los socios (Cavusgil et al., 2004). Por todo ello, Sahay (2003) reconoce que existe una relación importante entre la confianza y la colaboración y que la confianza tiene una influencia positiva sobre el compromiso y la mejora de la relación entre compradores y vendedores al por menor (Narayandas y Kasturi, 2004). Por ello, planteamos:

P2: La confianza favorece el aprendizaje organizacional en la cadena de suministro.

P3: La confianza favorece el compromiso en la cadena de suministro.

\subsubsection{Compromiso}

Otro de los factores que indican Selnes y Sallis (2003) para que haya aprendizaje organizacional entre los actores de la cadena de suministro es el compromiso. Según Monczka et al. (1998) cuando los socios comerciales están dispuestos a dedicar recursos para garantizar una interacción a largo plazo se obtiene éxito con una mayor probabilidad. Debido a que el compromiso no es más que la voluntad de un socio de dedicar tiempo, energía y recursos a la alianza (Spekman et al., 2002). De acuerdo con Anderson y Narus (1990), este compromiso incluye dedicar tiempo a la gestión de la alianza y realizar una inversión mayor, lo que incrementa la probabilidad de alcanzar acuerdos más exitosos. Ryu et al. (2009) añaden que se da compromiso cuando las empresas colaboran, comparten su información, su conocimiento y sus activos con sus socios lo que afecta positivamente el aprendizaje organizacional. Por lo que planteamos que: 


\section{P4: El compromiso favorece el aprendizaje organizacional en la cadena de suministro.}

\subsubsection{Sistemas tecnológicos}

Johnsen y Ford (2006) destacan dentro de las capacidades de interacción: los sistemas tecnológicos. Nos parece importante resaltar los factores tecnológicos debido al auge que han sufrido en los últimos años, según Clarke (1998) estamos asistiendo a una desagregación de información sin precedentes en el área de gestión de la cadena de suministro. Debido a los avances en la tecnología de las comunicaciones, y en Internet en particular, las empresas son capaces de utilizar estructuras sociales que se forman espontáneamente en los medios virtuales (Laine y Laine, 2012). Lusch et al. (2010) analizan la influencia de la tecnología de la información en la empresa, y destacan que a medida que aumenta la tecnología, se mejora la prestación de servicios, la capacidad de autoservicio, la capacidad de servir a los demás, de conocer a clientes y proveedores y la capacidad de interactuar con ellos. Todo esto conlleva que la capacidad para comunicarse a bajo coste aumente, así la coordinación entre empresas se vuelve más eficiente y sensible.

En la actualidad, las empresas están explorando plataformas como sitios web de redes sociales y foros de fans, estas fuentes tienen características novedosas, como la trascendencia de la demografía y la comunicación sin coste entre los participantes (Laine y Laine, 2012). La información adquirida a través de la red facilita que se dé una orientación hacia el mercado que según Narver y Slater (1990), integra a tres componentes, orientación al cliente, orientación al competidor y coordinación interfuncional. A su vez, la orientación al mercado se relaciona positivamente con la innovación (Grinstein, 2008). Uno de los inconvenientes de este aumento de información es que lleva a las empresas a verse desbordadas por el exceso de información. Además, se pueden tener grandes problemas si no se posee un sistema de gestión de información eficiente, lo que lleva a las organizaciones a obtener información directa sólo de aquello que necesitan (Huber, 1991).

Por otro lado, la gestión del conocimiento se puede definir como procesos organizacionales diseñados deliberadamente que rigen la creación, difusión, crecimiento y aprovechamiento de los conocimientos para cumplir con los objetivos organizacionales (Lee y Yang, 2000). Los sistemas de planificación de recursos empresariales son la columna vertebral de los sistemas de gestión de la cadena de suministro, incluyen bases de datos avanzadas que permiten a las empresas reunir información almacenada y recibida previamente de fuentes externas, para crear nuevos conocimientos para el intercambio de relaciones (Malhotra et al., 2005).

Las teorías sobre redes sociales han indicado que la unión y los vínculos relacionales pueden facilitar el intercambio de información y así contribuir al desarrollo de conductas innovadoras (Lee et al., 2008). Muchas empresas líderes en gestión de la cadena de suministro adoptan tecnología avanzada para permitir a los compradores y vendedores compartir conocimientos con mayor facilidad a través de las fronteras organizativas y de las fronteras nacionales (Kim et al., 2006; Myers y Cheung, 2008). En cadenas de suministro globales, las empresas han adoptado sistemas de tecnología de la información, que proporcionan la integración virtual entre los socios para coordinar su amplia gama de actividades en los canales, incluido el control de inventario y la planificación colaborativa y la previsión de negocio (Kim et al., 2006). La creciente integración ha hecho que las empresas de distribución, procesamiento y logística lleguen a depender en gran medida de la tecnología de información para transferir grandes cantidades de datos e información en toda la cadena de suministro (Hyland et al., 2003). 
Sin embargo, todavía son muchas las empresas reacias a adoptar estos sistemas de tecnología de la información, por las características específicas de su organización o bien por el miedo a implantar nuevos sistemas que pongan en peligro su status quo. Por eso podemos hablar de dos tipos de empresas distintas que distinguimos por la cultura que tienen en relación a la tecnología. Estos tipos de culturas pueden ser de bajo o alto contexto, según la mayor parte de la información está en el contexto físico e interiorizado en la persona, o aquellas culturas en las que la información está explícita en el mensaje, respectivamente. En una cultura de bajo contexto, las empresas confían más en las bases de datos e Internet para llevar a cabo la comunicación explícita (Jean et al., 2010). En las de contexto alto la gente confía más en el contacto humano y las relaciones personales. La innovación tecnológica es menos probable que sea adoptada y aceptada en una cultura de alto contexto (Van Everdingen y Waarts, 2003), ya que existe incertidumbre tecnológica, entendiendo por la misma el grado de volatilidad, de cambio, e imprevisibilidad de la tecnología en la relación cliente-proveedor (Lee et al., 2008).

Para finalizar con el análisis de los factores tecnológicos en el aprendizaje organizacional, nos gustaría hacer referencia al concepto introducido por Lusch et al. (2010), que en su estudio nos hablan de un ecosistema de servicios. Entendiendo por tal una estructura espacial y temporal espontánea de detección y respuesta de valor propuesta por actores sociales y económicos que interactúan a través de las instituciones y de la tecnología, para coproducir ofertas de servicios, intercambios de ofertas de servicios y co-creación de valor. Este ecosistema también puede ser visto como una red de valor, que está formado principalmente por lazos débiles (Granovetter, 1973), que permiten a las organizaciones de redes formar una macroestructura que pueda ser más fluida, ágil y adaptable (Lusch et al., 2010). Por ello planteamos que:

P5: Una mayor comunicación e interacción de la empresa a través de Internet con sus socios de la cadena de suministro aumenta la capacidad de aprendizaje organizacional de la empresa.

\subsection{Factores internos que explican el aprendizaje organizacional en la cadena de suministro}

\subsubsection{Cultura de la cadena de suministro}

En una organización, una cultura fuerte y una asociación común pueden llevar a los miembros a compartir propósitos/objetivos (Gioia y Thomas, 1996) y, con ello, dar lugar a un mayor flujo de conocimiento. Los recursos compartidos de la cadena de suministro pueden sustituir a las características tradicionales que unen a los miembros de una empresa, tales como la cultura (Weick, 1987). Es por ello que hoy en día conceptos como la calidad y la puntualidad de la cadena de suministro son imprescindibles para que la atención se centre en las actividades necesarias (Handfield y Nichols, 2002).

En un mercado que se caracteriza por diferencias culturales y complejidad de productos, la relación de aprendizaje es difícil de lograr y depende en gran medida de una estrecha colaboración entre los socios, como es el ejemplo de la cadena internacional de electrónica (Jean et al., 2010). Griffith et al. (2006) han indicado que las diferencias entre las culturas nacionales de cadenas de suministro con socios internacionales pueden influir en el aprendizaje y el conocimiento compartido. Por lo tanto, vamos a analizar también la cultura de la cadena de suministro como factor que influye en su aprendizaje organizacional. 
La cultura refleja las normas centrales que caracterizan a una organización y dan forma a las expectativas acerca de lo que son comportamientos apropiados y actitudes (Schwartz y Davis, 1981). Una cultura que apoya la confianza, la apertura, la investigación y la experimentación es probable que obtenga más beneficios de las alianzas de aprendizaje que la que evita estas alianzas, debido al riesgo potencial asociado con la transferencia de conocimiento (Spekman et al., 2002). Así, una orientación empresarial se identifica porque los miembros de la cadena de suministro están asociados a valores de búsqueda de nuevas oportunidades de mercado y de renovación de las áreas existentes de las actividades de la cadena de suministro (Naman y Slevin, 1993) alcanzando así una cultura de competitividad.

La cultura de competitividad se puede definir como el grado en que las cadenas de suministros están dispuestas a detectar y llenar los vacíos entre lo que el mercado desea y lo que ofrece (Hult et al., 2002) y es reflejado por tres orientaciones: innovación, emprendimiento y aprendizaje (Godfrey y Hill, 1995). La cultura de competencia ofrece a los miembros de la cadena de suministro un patrón de valores y creencias que reafirman la importancia de estos elementos (Hult et al., 2007). Estas culturas de competitividad son un recurso estratégico valioso, raro e inimitable en las cadenas de suministro (Wernerfelt, 1984) que proporcionan una ventaja competitiva sostenible.

La cultura competitiva, el desarrollo de conocimiento y la interacción entre ambas tienen un efecto positivo en el rendimiento del tiempo de suministro o abastecimiento de la cadena de suministro según Hult et al. (2007), quienes explican el tiempo del suministro como la longitud de tiempo necesario entre la toma de un pedido y la entrega del producto para el cliente. El tiempo de suministro de una cadena de suministro es un indicador clave que permite evaluar directamente su funcionamiento (Nichols et al., 1996), además de ser fundamental para el éxito estratégico de la empresa (Hult et al., 2007). La idea de aprendizaje organizacional proporcionan la base para predecir un vínculo entre las actividades de distribución de información y el tiempo de suministro (Hult et al., 2002).

Un ejemplo para entender todo esto lo encontramos cuando nos referimos a la excelencia en el tiempo de abastecimiento. Es decir, que sea lo más corto posible, lo que permite a las empresas crecer más rápido y obtener beneficios más altos en relación a otras empresas de su sector, aumentar la cuota de mercado a través de la introducción temprana de nuevos productos, controlar los gastos generales, los costes de inventario y moverse con posiciones de liderazgo en la industria (Handfield y Nichols, 2002). Este aumento de habilidades y conciencia, debe promover la capacidad de minimizar el tiempo del ciclo de la cadena de suministro según Huber (1991). Además, la reducción del tiempo de ciclo de la cadena es un fuerte vínculo entre sus socios, lo que sugiere que los miembros principales que piensan igual acerca de conceptos tales como la calidad y la velocidad pueden enfatizar el intercambio de información y los debates cara a cara, mejorando así el rendimiento de la cadena de suministro (Hult et al., 2004). Es por todo ello que planteamos que:

P6: Una cultura de competencia favorece el aprendizaje organizacional en la cadena de suministro.

\subsubsection{Orientación al mercado/cliente}

El aprendizaje organizacional es inseparable de una orientación al mercado, puesto que el aprendizaje es una característica cultural de la organización que se ocupa de la comercialización y demandas de los clientes (Hurley y Hult, 1998). Vieira (2010) reveló que la orientación al mercado tiene un fuerte impacto en el aprendizaje organizacional y que se relacionan positivamente. La orientación al mercado contribuye al intercambio de 
información en la cadena de suministro, por lo que se considera parte de la práctica de aprendizaje organizacional. Además, una orientación al mercado ayuda a una empresa para obtener aprendizaje organizacional de otras empresas (Min y Mentzer, 2000). Por lo que, la orientación al mercado, según Narver y Slater (1990), la orientación al cliente, la orientación al competidor y la coordinación inter-funcional afectan positivamente la estrategia de gestión de la cadena de suministro.

Low et al. (2007) defienden que altos niveles de la orientación al mercado conducen a altos niveles de innovación dentro de una empresa y la innovación o capacidad de innovar de ésta tiene relación con el desempeño de la misma. Aquellas empresas que están más preocupadas en satisfacer las necesidades de los consumidores son las primeras en llevar a cabo innovaciones dentro de la empresa en cualquiera de las dimensiones posibles (innovación de productos, innovación de procesos, la innovación administrativa, innovación de marketing e innovación de servicios). Según Lin et al. (2010), la innovación y la orientación al mercado están relacionadas, consiguiendo un mayor desempeño de la empresa. Además, expusieron que la gestión basada en la orientación al mercado se consideraba fundamental en la mejora de la innovación de la empresa.

En resumen, la estrategia de gestión de la cadena de suministro, el aprendizaje organizacional, la innovación en la cadena de suministro, la confianza, el compromiso y la colaboración juegan un papel de mediación en la relación entre la orientación al mercado y el desempeño de la cadena de suministro (Tukamuhabwa et al., 2011). Min y Mentzer (2000) estudiaron la relación entre el marketing y la gestión de la cadena de suministro y establecieron que la orientación al mercado juega un papel fundamental en la gestión de esta. Una cultura de mejora continua da lugar a una cultura de la innovación, esto tiene gran importancia si se considera que el desarrollo de una cultura de la innovación es fundamental para la capacidad de las empresas para desarrollar y adoptar nuevas orientaciones estratégicas, mientras que la mejora continua sólo permite a una empresa tener más éxito en la búsqueda de una estrategia específica o un conjunto de objetivos (Hyland et al., 2003). Planteamos que:

P7: Una orientación al mercado favorece el aprendizaje organizacional en la cadena de suministro.

\subsubsection{Innovación}

Hoy en día es sumamente importante para las empresas ser innovadoras, Hurley y Hult (1998) consideran la innovación o la capacidad de innovación como una capacidad que mejora la competitividad y el rendimiento. Esta capacidad de innovar lleva a las empresas a estar en constante interacción con el mercado, en la búsqueda de nuevas ideas. De hecho, tener una orientación hacia la innovación se refiere a facilitar a los miembros de la cadena de suministro valores asociados con la generación de nuevas ideas (Hurley y Hult, 1998), esto también se refiere a la utilización o creación de tecnología aplicada a los sistemas, políticas, programas, productos, procesos, dispositivos o servicios que son nuevos en una organización (Chang y Lee, 2008).

Recientemente Chesbrough (2003), ha desarrollado un nuevo enfoque de innovación que tiene una gran importancia en el ámbito de la investigación. Este enfoque de innovación abierta promueve la apertura de los límites de la empresa, lo que permite que el conocimiento fluya dentro y fuera de la empresa (Chesbrough, 2003), estos flujos de conocimiento afectan al capital intelectual de la empresa, en sí mismo son un recurso decisivo para alcanzar la innovación (Laine y Laine, 2012). El marco de la innovación abierta también incluye salidas de conocimiento a través de la misma frontera. Esto significa que el conocimiento de una 
empresa se utiliza fuera de ella buscando nuevos usos, mercados y modelos de negocio. Por ejemplo, la concesión de licencias, patentes de la empresa, creación de spin-off de innovaciones y tecnologías que no contribuían al negocio principal de la empresa (Laine y Laine, 2012).

En nuestro estudio, sin lugar a dudas, nos interesa este marco de innovación abierta debido a que no podemos entender la empresa como un ente cerrado que no transfiera información y conocimiento más allá de los límites de la empresa. Para nosotros es imprescindible relacionar la innovación con los flujos de conocimiento externos a la organización y con el aprendizaje organizacional. Una de las relaciones que se da entre innovación y aprendizaje es según el grado de radicalidad o incrementalidad de la innovación, en este sentido, las innovaciones más radicales logran una cantidad mayor de aprendizaje (Gupta et al., 2006), a su vez, un mayor número de innovaciones contribuyen al conocimiento de la organización que se ayuda en la generación de más combinaciones de conocimiento en un círculo vicioso.

La orientación a la innovación tiene una asociación positiva con el desarrollo del conocimiento en la cadena de suministro (Hult et al., 2007). La orientación a la innovación se refiere a los valores que tiene un proveedor en relación con la generación de nuevas ideas en la relación cliente-proveedor transfronteriza (Hult et al., 2004). Las empresas con mayor capacidad innovadora pueden alcanzar un mayor grado de aprendizaje entre empresas (Simonin, 2004), la innovación es una de las capacidades organizativas más importantes vinculadas al éxito la empresa (Hult et al., 2004). En este sentido la orientación a la innovación y la orientación estratégica de organización han sido vistas como culturas que pueden poner a empresas delante de su competencia (Hult et al., 2007; Siguaw et al., 2006). Si tenemos en cuenta la innovación que se da dentro de la cadena de suministro podemos resaltar los beneficios que estudió Stundza (2009). Según este autor, la innovación de la cadena de suministro provoca eficiencia dentro de ésta, como la reducción del tiempo de espera, las nuevas estrategias de operación, reducción de costes, el suministro de una calidad constante y el desarrollo de la flexibilidad para hacer frente a los cambios rápidos en el entorno empresarial.

Uno de los motivos por los que nos centramos en la cadena de suministro es porque se ha demostrado recientemente que las empresas emprendedoras tienden a ser más pequeñas, más innovadoras y más ágiles que sus contrapartes más grandes (Gray y McNaughton, 2010), por lo tanto, las emprendedoras suelen centrarse más en la búsqueda de nuevas ideas que en el desarrollo de los procesos actuales, con la consiguiente externalización de fases de la cadena de valor de la empresa. Este tipo de empresas suelen tener poca integración vertical y un gran número de proveedores.

La relación con los proveedores toma importancia en la innovación propia, concretamente debido a la innovación de proveedores, que afecta directamente en la innovación y el rendimiento de la empresa que lo subcontrata. Los atributos de la innovación del proveedor, como el tipo de innovación (producto o proceso), o el grado de incrementalidad, o radicalidad del diseño pueden moderar el efecto de la innovación en el rendimiento fabricante (Meyers et al., 1999; Tidd, 1995). La innovación del proveedor puede mejorar las capacidades del fabricante (Freeman y Cavinato, 1990), ya sean en forma de nuevos productos, nuevos métodos de producción, nuevos mercados, nuevas fuentes de abastecimiento, o de nuevos procesos de negocio (Schumpeter, 1934), afectando así al rendimiento del cliente de fabricación. 
La capacidad de la empresa para crear innovaciones puede promulgar aprendizaje útil por el fabricante como proveedor de innovación (Hult et al., 2004), este aprendizaje interorganizacional hace que el comprador puede beneficiarse no sólo directamente de la innovación, sino también indirectamente a través de la transferencia de conocimiento (Hamel, 1991). Debido a esto, la estrategia de abastecimiento debe tener en cuenta la capacidad de innovación del proveedor (Handfield y Nichols 2002) ya que las innovaciones en los procesos de un proveedor puede conducir a la reducción de los costes de producción para el fabricante que utiliza sus productos (Kim, 2000). innovar.

P8: Las empresas que tienen mayor aprendizaje organizacional son más propensas a

P9: Las empresas que realizan innovaciones en su organización fomentan el aprendizaje organizacional.

En la figura 1, se recogen todas las relaciones planteadas.

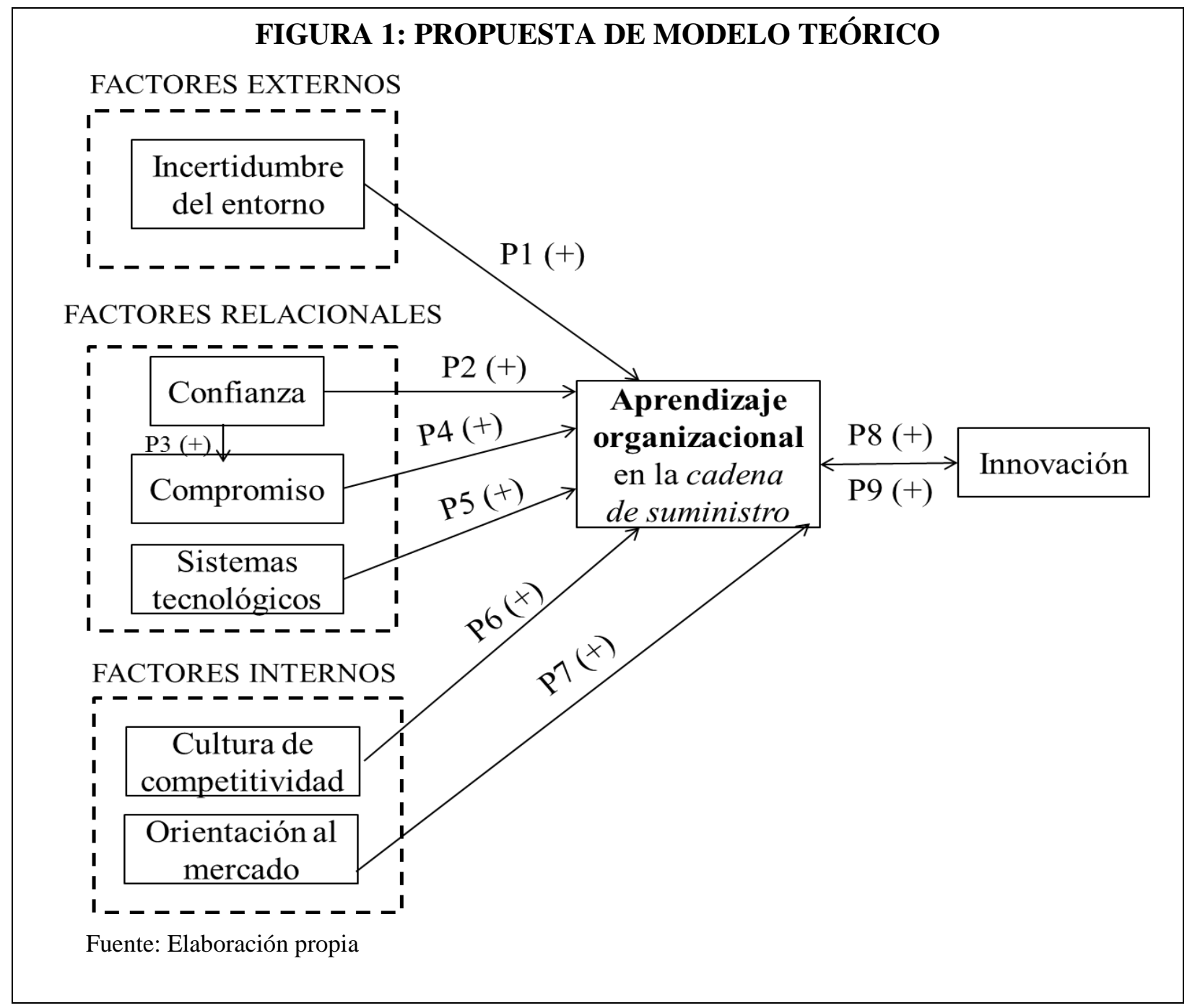

\section{CONCLUSIONES}

Las cadenas de suministro no pueden verse como simples mecanismos de transferencia y suministro de productos que conllevan un ahorro en costes transaccionales, sino como un conjunto de empresas interdependientes que colaboran juntas en pro de una mayor 
competitividad. Una cadena de suministro es vista como un sistema de procesamiento, no sólo de materiales, sino también de información. En la elección de proveedores deben tenerse en cuenta unas nuevas aptitudes como la capacidad de innovación y la búsqueda de una visión común.

El flujo de información y conocimientos que transfieren las empresas dentro de la cadena de suministro favorece que sean más ágiles, más competitivas y puedan aprender mutuamente. El aprendizaje organizacional entre las empresas es una capacidad dinámica que puede proveer a las empresas de una ventaja competitiva conjunta. Pero como consecuencia de los recientes cambios en el mercado, las empresas se ven obligadas a llegar a acuerdos de colaboración con otras empresas y depender de los miembros de la cadena de suministro para ser competitivos. Esta dependencia crea cierta desconfianza hacia las otras empresas, lo que le lleva a proteger su conjunto de capacidades y no compartir información confidencial con sus proveedores. Romper esta barrera de desconfianza y miedo al oportunismo supone un gran reto para las empresas.

Podemos dividir los principales factores que inciden en el aprendizaje organizacional de la cadena de suministro en las siguientes categorías: a) factores externos: incertidumbre del entorno, b) factores relacionales: confianza, compromiso y sistemas tecnológicos y c) factores internos: cultura, orientación al mercado e innovación. Conocer estos factores nos permite analizarlos en futuros trabajos y buscar cómo actuar para disuadir los comportamientos hostiles en compartir información con los socios de la cadena de suministro. Se deben romper esas barreras psicológicas que, por miedo al oportunismo, han impedido colaborar eficientemente con los proveedores/clientes y transferir información para incentivar el aprendizaje organizacional.

\section{Futuras líneas de investigación}

Después del análisis teórico realizado proponemos algunas líneas de investigación para futuros trabajos académicos. Por ejemplo, ampliar el número de variables analizadas que influyen en el aprendizaje organizacional de la cadena de suministro. Así como, desarrollar las proposiciones planteadas en el presente trabajo y poder comprobar empíricamente si serían aceptadas o no. Por otro lado, sería interesante profundizar en el concepto de ecosistemas de servicios y relacionarlo con el aprendizaje organizacional de la cadena de suministro y estudiar cómo minorar la desconfianza que hay entre los socios de la cadena de suministro para así fomentar el aprendizaje mutuo.

\section{BIBLIOGRAFÍA}

Anderson, J. y Narus, J. (1990): "A model of distributor firm and manufacturer firm working partnerships", Journal of Marketing, 48, 62 - 74.

Baker, W.E. y Sinkula, J.M. (1999): "The synergistic effect of market orientation and learning orientation on organizational performance", Journal of the Academy of Marketing Science, 27: $411-427$.

Bowersox, D.J., Closs, D.J. y Stank, T.P. (1999): 21st Century Logistics: Making Supply Chain Integration a Reality, Council of Logistics Management (CLM), Chicago, Il.

Brown, J.E. y Hendry, C. (1997): "Industrial districts and supply chain as vehicles for managerial and organizational learning", International Studies of Management \& Organization, White Plains", 27, 127 - 58.

Brown, J.S. y Duguid, P. (2001): "Knowledge and Organization: A Social-Practice Perspective", Organization Science, 12(2), 198 - 213. 
Cavusgil, S. Tamer, Deligonul, S. y Zhang, C. (2004): "Curbing Foreign Distributor Opportunism: An examination of Trust, Contracts, and the Legal Environment in International Channel Relationships", Journal of International Marketing, 12, 7 - 27.

Chang, S. y Lee, M. (2008): "The linkage between knowledge accumulation capability and organizational innovation", Journal of Knowledge Management, 12(1), 3 - 20.

Chesbrough, H.W. (2003): Open innovation: the new imperative for creating and profiting from technology, Boston, MA: Harvard Business School Press.

Chow, W.S., Madu, C. N, Kuei, C-H., Lu, M.H., Lin, C. y Tseng. H. (2008): "Supply chain management in the US and Taiwan: An empirical study", The International Journal of Management Science, 36, 665-679.

Clarke, M.P. (1998): "Virtual Logistics: An Introduction and Overview of the Concepts", International Journal of Physical Distribution \& Logistics Management, 28(7), 486 507.

Cohen, W.M. y Levinthal, D.A. (1990): "Absorptive capacity: a new perspective on learning and innovation", Administrative Science Quarterly, 35, 128 - 52.

Cooper, M.C. y Ellram, L.M. (1993): "Characteristics of supply chain management and the implications for purchasing and logistic strategy", The International Journal of Logistics Management, 4, 13 - 24.

Corner, P.D., Kinicki, A.J. y Keats, B.W. (1994): "Integrating organizational and individual information processing perspectives on choice”, Organization Science, 5(3), 294 308.

Crossan, M. y Inkpen, A. (1995): “The subtle art of learning through alliances", Business Quarterly, 60(2), 68.

Crossan, M.M., Lane, H.W. y White, R.E. (1999): “An organizational learning framework: from intuition to institution", Academy of Management Review, 24(3), 522 - 537.

Daft, R.L. y Weick, K.E. (1984): "Toward a model of organizations as interpretation systems", Academy of Management Review, 9, (2), 284-295.

Dainty, A.R.J. y Brooke, R.J. (2004): "Towards improved construction waste minimization: a need for improved supply chain integration?", Structural survey, 22: 20 - 9.

Dong, J.Q. y Yang, C.H. (2015): "Information technology and organizational learning in knowledge alliances and networks: Evidence from U.S. pharmaceutical industry", Information \& Management, 52(1), 111-122.

Dyer, J. y Nobeoka, K. (2000): "Creating and Managing a High Performance KnowledgeSharing Network: The Toyota Case", Strategic Management Journal, 21: 345 - 367.

Easterby-Smith, M. (1997): "Disciplines of Organizational Learning: Contributions and Critiques", Human Relations, 50(9), 1085 - 1114.

Eisenhardt, K. y Martin, J. (2000): “Dynamic Capabilities: What Are They?", Strategic Management Journal, 21(10-11), 1105 - 1121.

Everdingen, Y.M. y Van, Waarts, E. (2003): "The effect of national culture on the adoption of innovations", Marketing Letters, 14, (3), 217-232.

Fiol, C.M. y Lyles, M. (1985): “Organizational Learning”, The Academy of Management Review, 10(4), 803 - 813.

Freeman, V.T. y Cavinato, J.L. (1990): "Fitting Purchasing to the Strategic Firm: Framework, Processes, and Values", Journal of Purchasing and Materials Management, 26(1), 6 10. 
Gioia, D.A. y Thomas, J.B. (1996): "Identity, image, and issue interpretation: sensemaking during strategic change in academia”, Administrative Science Quarterly, 41, 370 403.

Godfrey, P.C. y Hill, C.W.L. (1995): "The problem of unobservable in strategic management research", Strategic Management Journal, 16(7), 519 - 533.

Goes, J.B. y Park, S.H. (1997): "Interorganizational Links and Innovation: The Case of Hospital Services”, Academy of Management Journal, 40(3), 673 - 696.

Goffin, K., Szwejczewski, M. y New, C. (1997): "Managing suppliers: when fewer can mean more", International Journal of Physical Distribution \& Logistic Management, 27: $422-7$.

Granovetter, M.S. (1973): “The Strength of Weak Ties”, The American Journal of Sociology, 1360-1380.

Grant, R.M. (1996): “Toward a knowledge-based theory of the firm", Strategic Management Journal, 17, 109 - 122.

Grantham, C.E., Nichols, L.D. y Schonberner, M. (1997): "A framework for the management of intellectual capital in the healthcare industry", Journal of Health Care Finance, 33, $1-19$.

Gray, B. y McNaughton, R. (2010): "Knowledge, values, and InternationalisationIntroduction to the Special Edition", Journal of International Entrepreneurship, 8(2), $115-120$.

Green, Jr., Kenneth, W., McGaughey, R. y Casey, M. (2006): "Does supply chain management strategy meadiate the association between market orientation and organizational performance?", Supply Chain Management: An International Journal, 11(5), $407-414$.

Griffith, D., Cavusgil, S. y Xu, S. (2008): "Emerging Themes in International Business Research", Journal of International Business Studies, 39(7), 1220 - 1135.

Griffith, D., Myers, M. y Harvey, M. (2006): “An Investigation of National Culture's Influence on Relationship and Knowledge Resource in Interorganizational Relationship between Japan and the United States", Journal of International Marketing, 14(3), 1 - 32 .

Grinstein, A. (2008): "The effect of market orientation and its components on innovation consequences: a meta-analysis", Journal of Academy of Marketing Science, 36, 166 173.

Gulati, R. (1995): "Does familiarity breed trust? The implications of repeated ties of contractual choice in alliances", Academy of Management Journal, 38, 85 - 112.

Gupta, A.K., Smith, K.G. y Shalley, C.E. (2006): "The interplay between exploration and explotation", Academy of Management Journal, 49(4), 693 - 706.

Hagedoorn, J. (1993): "Undertanding the rationale of strategic technology partnering: Interorganizational modes of cooperation and sectorial differences", Strategic Management Journal, 14(5), 371.

Hall, R. (1999): "Rearranging risk and rewards in supply chain management", Journal of General Management, 24, 22 - 23.

Hamel, G. (1991): "Competition for competence and inter-partner learning within international strategic alliances", Strategic Management Journal, 12, 83 - 103. 
Handfield, R.B. y Nichols, E.L. (2002): Supply Chain Redesign: Transforming Design Chains into Integrated Value System, Financial Times-Prentice-Hall: Upper Saddle River, NJ.

Harhoff, D. (1996): "Strategic Spillovers and Incentives for Research and Development", Management Science, 42(6), 907 - 926.

Hendricks, K.B. y Singhal, V.R. (2003): "The effect of supply chain glitches on shareholder wealth", Journal of Operations Management, 21(5), 501 - 522.

Hernández, E.M., Rodríguez, O.A. y Sánchez, P.M. (2010). "Inter-organizational governance, learning and performance in supply chains", Supply Chain Management: An International Journal, 15(2), 101 - 114.

Huber G.P. (1991): "Organizational learning: the contributing processes and the literatures", Organization Science, 2(1), 88 - 115.

Hult, G.T., Ferrell, O.C. y Schul, P. (1998): “The effect of global leadership on purchasing process outcomes", European Journal of Marketing, 32, 11 - 12.

Hult, G.T., Ketchen, D.J. y Arrfelt, M. (2007): "Strategic supply chain management: Improving performance through a culture of competitiveness and knowledge development”, Strategic Management Journal, 28, 1035 - 1052.

Hult, G.T., Ketchen, D.J. y Slater, S.F. (2004): "Information processing, knowledge development, and strategic supply chain performance", Academy of Management Journal, 47 (2), $241-253$.

Hult, G.T., Ketchen, D.J. y Nichols, E.L. (2002): “An examination of cultural competitiveness and order fulfillment cycle time within supply chains", Academy of Management Journal, 45, $501-511$.

Hurley, R.F. y Hult, G.T. (1998): "Innovation, market orientation, and organizational learning: an integration and empirical examination", Journal of Marketing, 62(3), 42 54.

Hyland, P.W., Soosay, C. y Sloan, T. (2003): "Continuos improvement and learning in the supply chain", International Journal of Physical Distribution \& Logistic Management, 33(4), 316 - 335.

Jean, R., Sinkovics, R. y Kim, D. (2010): "Drivers and Performance Outcomes of Relationship Learning for Suppliers in Cross-Border Customer-Supplier Relationships: The Role of Communication Culture", Journal of International Marketing, 18(1), 63 - 85

Johnsen, R. y Ford, D. (2006): "Interaction Capability Development of Smaller Suppliers in Relationships with Larger Customers", Industrial Marketing Management, 35(8), $1002-1015$.

Juttner, U., Christopher, M. y Godsell, J. (2010): "A strategic framework for integrating marketing and supply chain strategies", The international Journal of Logistics Management, 21(1), $104-12$.

Kim, B. (2000): "Coordinating an Innovation in Supply chain Management", European Journal of Operational Research, 123(3), 658 - 584.

Kim, D., Cavusgil, T. y Calantone, R. (2006): "Information system Innovations and Supply Chain Management: Chanel Relationships and Firm Performance", Academy of Marketing Science Journal, 34(1), 40 - 54. 
Knowles G., Whicker, L., Heraldez, J.F. y Del Campo, C.F. (2005): "A conceptual model for the application of six sigma methodologies to supply chain improvement", International journal of logistics: research and applications, 8, (1), 51-65.

Kohli, A.K., Jaworski, B.J. y Kumar, A. (1993): "MARKOR: a measure of market orientation", Journal of Marketing Research 15, 467 - 477.

Kuei, C., Madu, C. N., Chow, W. S. y Lu, M. H. (2005): "Supply chain quality and excellence in the new economy: an empirical study of Hong Kong based firms", Multinational Business Review, Vol. 13, No. 1, pp.33-53.

Kumaraswamy, M.M., Anvuur, A. y Mahesh, G. (2008): Contractual frameworks and cooperative relationships. Collaborative Relationships in Construction Developing Frameworks \& Network, Wiley-Blackwell, Oxford. 78 - 104.

Laine, M. y Laine, A. (2012): "Open Innovation, Intellectual Capital and Different Knowledge Sources", European Conference on Intellectual Capital, 12, 239-241.

Lane, P.J. y Koka, B.R. (2006): "The reification of Absorptive Capacity: A Critical Review and Rejuvenation of the Construct", Academy of Management Review, 31(4), 833 863.

Lee, A., Chen, H. y Tang, Y. (2008): "Developing new products in a network with efficiency and innovation”, International Journal of Production Research, 46(17), 4687 - 4707.

Lee, C.C. y Yang, J. (2000): "Knowledge Value Chain", Journal of Management Development, 9, $783-93$.

Lei, D., Slocum, J.W. y Pitts, R.A. (1997): "Building cooperative advantage: managing strategic alliances to promote organizational learning", Journal of World Business, 32, $203-224$.

Lennon, A. y Wollin, A. (2001): "Learning organisations: Empirically investigating metaphors", Journal of Intellectual Capital, 2(4), 401 - 422.

Levinson, N.S. y Asahi, M. (1995): "Cross-National Alliances and Interorganizational Learning", Organizational Dynamics, 24(2), 50-63.

Levinthal, D. y March, J.G. (1981): "A model of adaptive organizational search", Journal of Economic Behavior and Organization, 2(4), 307 - 333.

Levitt, B. y March, J.G. (1988): “Organizational Learning”, Annual Review of Sociology, $14(1), 319-340$.

Lewis, E. (2005): "Now is SMEs time to compete on-demand supply chain solutions are affordable for the small distributor", Industrial Distribution, September.

Lin, R., Chen, R. y Kevin, C. (2010): "Customer relationship management and innovation capability: an empirical study", Industrial Management \& Data System, 110(1), 111 133.

Low, D. R., Chapman, R.L. y Sloan, T. (2007): "Inter relationships between innovation and market orientation in SMEs", Management Research News, 30(12), 878 - 891.

Lummus, R. y Vokurka, R.J. (1999): "Defining supply chain management: a historical perspective and practical guidelines", Industrial Management and Data Systems, 99, $11-17$.

Lusch, R. (2011): "Reframing Supply Chain Management: A Service-Dominant Logic Perspective", Journal of Supply Chain Management, 41(1), 14 - 18.

Lusch, R.F., Vago, S.L. y Tanniru, M. (2010): "Service, Value Networks and Learning", Journal of the Academy of Marketing Science, 38, 19 - 31. 
Malhotra, A., Gosain, S. y El Sawy, O. (2005): "Absorptive capacity configurations in supply chains: gearing for partner enabled market knowledge creation”, MIS Quarterly, 29(1), $145-187$.

Maloni, M.J. y Benton, W.C. (1997): "Supply chain partnership: opportunities for operations research", European Journal of Operations Research, 419-29.

Martínez-Costa, M. y Jiménez-Jiménez, D. (2009): "The effectiveness of TQM: the key role of organizational learning in small businesses", International Small Business Journal, $27,98-125$.

Meyers, P.W., Sivakumar, K. y Nakata, C. (1999): "Implementation of Industrial Process Innovations: Factors, Effects and Marketing Implications", The Journal of Product Innovation Management, 16(3), 295 - 311.

Min, S. y Mentzer, J. (2000): "The role of marketing in supply chain management. International", Journal of Physical Distribution Management, 30(9), 765 - 787.

Monczka, R.M., Petersen, K.J., Handfield, RB. y Ragatz, GL. (1998): "Success factors in strategic supplier alliances: the buying company perspective", Decision Sciences, 29, $553-577$.

Myers, M. y Cheung, M. (2008): "Sharing Global Supply Chain knowledge", MIT Sloan Management Review, 49(4), $67-73$.

Naman, J.L. y Slevin, D.P. (1993): "Entrepreneurship and the concept of fit: a model and empirical test", Strategic Management Journal, 14(2), 137 - 154.

Narasimhan, R. y Das, A. (1999): “An Empirical Investigation of the Contribution of Strategic Sourcing to Manufacturing Flexibilities and Performance", Decision Sciences, 30, 683-718.

Narayandas, D. y Kasturi, R.V. (2004): "Building and Sustaining Buyer - seller Relationships in Mature Industrial Markets", Journal of Marketing , 68, 63 - 77.

Narver, C.J. y Slater, F.S. (1990): "The effect of a marketing orientation on business profitability", Journal of Marketing, 54, 20 - 35.

Nelson, R.R. y Winter, S.G. (1973): "Toward an Evolutionary Theory of Economic Capabilities", American Economic Review (Papers and Proceedings), 68, 440-449.

Nichols, E.L., Retzlaff, R. D. y Florick, M.N. (1996): "Reducing order fulfillment cycle time in an international supply chain", Cycle Time Research, 2(1), 13 - 28.

O’Laughlin, K. (2000): “High Performance Value Chains”, Cap Gemini Ernst \& Young, New York, NY.

Park, S. y Hartley, J. (2002): "Exploring the effect of supplier management on performance in the Korean automotive supply chain", Journal of Supply Chain Management, 38(2), $46-52$.

Penrose, E. (1959): The theory of the growth of the firm. Wiley, New York.

Popper, M. y Lipshitz, R. (2000): “Organizational Learning: Mechanisms, Culture, and Feasibility", Management Learning, 31(2), 181 - 196.

Power, D. (2005): "Supply chain management integration and implementation: a literature review", Supply Chain Management: An International Journal, 10, 252 - 263.

Quinn, J.B. (1999): "Strategic outsourcing: leveraging knowledge opportunities", Sloan Management Review, Summer, 9-21. 
Roh, J.J., Hong, P. y Park, Y. (2008): "Organizational culture and supply chain strategy: a framework for effective information flows", Journal of Enterprise Information Management, 21(4), $361-376$.

Roth, A., Marucheck, A., Kemp, A. y Trimble, D. (1994): "The Kowledge factory for accelerated learning practices". Planning Review, 22(3), 26.

Ryu, I., Soon, H. y Chulmo, J. (2009): "The role of partnership in supply chain performance", Industrial Management \& data Systems, 109(4), 496 - 514.

Sahay, B.S. (2003): "Understanding trust in supply chain relationships", Industrial Management \& Data Systems, 103(8), 553 - 563.

Schein, E.H. (1984): "Coming to a new awareness of organizational culture", MIT Sloan Management Review, 25, 3 - 15.

Schumpeter, J.A. (1934): “The Theory of Economic Development”, Cambridge, MA, Harvard University Press.

Schwartz, H. y Davis, S. (1981): "Matching Corporate Culture and Business Strategy," Organizational Dynamics, pp. 30- 48.

Selnes, F. y Sallis, J. (2003): "Promoting relationship learning”, Journal of Marketing, 67(3), 80.

Siguaw, J., Simpson, P. y Enz, C. (2006): "Conceptualizing Innovation Orientation: A Framework for Study and Integration of Innovation Research", The Journal of Product Innovation Management, 23(6), 556 - 574.

Simonin, B. (2004): "An empirical investigation of the Process of Knowledge Trnasfer in International Strategic Alliances", Journal of International Business Studies, 35(5), $407-427$.

Sinkula, J., Baker, W. y Noordewier, T. (1997): "A framework of market-based organizational learning: linking value, knowledge and behavior", Academy of Marketing Science Journal, 25(4), 305 - 318.

Slater, S.F. y Narver, J.C. (1995): "Market orientation and the learning organization", Journal of Marketing, 59(3), 63 - 74.

Spekman, R.E., Kamauff, J.W. y Myhr, N. (1998): “An empirical investigation into supply chain management: a perspective on partnerships", International Journal of Physical distribution \& Logistics Management, 28, 630 - 650.

Spekman, R.E., Spear, J. y Kamauff, J. (2002): "Supply chain competency: learning as a key component”, Supply Chain Management, 7(1).

Stuart T. y Scott F. (2013): "Organizational learning in construction supply chains", Engineering, Construction and Architectural Management", 20, (1), 83-98.

Stundza, T. (2009): "Smart sourcing summit provides supply risk management tips", Purchasing, 138(11), 15.

Styhre, A., Josephson, P. y Knauseder, I. (2004): "Learning capabilities in organizational networks: case studies of six construction projects", Construction Management and Economics, November, 22, 957-966.

Subramani, M. y Venkatraman, N. (2003): "Safeguarding Investments in Asymmetric Interorganizational Relationships: Theory and Evidence". Academy of Management Journal, 46(1), 46 - 62 . 
Subramaniam, M. y Youndt, M.A. (2005): "The influence of intellectual capital on the types of innovative capabilities", Academy of Management Journal, 48, 450 - 463.

Teece, D., Pisano, G., y Shuen, A. (1997): "Dynamic Capabilities and Strategic Management", Strategic Management Journal, 18(7), 509 - 533.

The Logistics and Distribution Institute. (2000): Web page, available at: www.louisville.edu/org/lodi/About.html, University of Louisville.

Tidd, J. (1995): "Development of Novel Products through Intraorganizational and Interorganizational Networks the Case of Home Automation", Journal of Product Innovation Management, 12(4), 307 - 322.

Tsai, Y. (2001): "Comparative analysis of model management and relational database management", Omega, 29(2), 157 - 179.

Tu, Q., Vonderembse, M.A., Ragu-Nathan, T.S. y Sharkey, T.W. (2006): "Absortive Capacity: Enhancing the Assimilation of Time-Based Manufacturing Practices", Journal of Operation Management, 24(5), 692 - 710.

Tukamuhabwa, B., Eyaa, S. y Derek, F. (2011): "Mediating Variables in the Relationship between Market Orientation and Supply Chain Performance: A Theoretical Approach", International Journal of Business and Social Science, 2(22), 63 - 85.

Udin, Z.M., Khan, M.K. y Zairi, M. (2006): "A collaborative supply chain management framework Part 1 - planning stage", Business Process Management Journal, 12, (3), 361-376.

Van Everdingen, Y.M. y Waarts, E. (2003): "The Effect of National Culture on the Adoption of Innovations", Marketing Letters, 14 (3), 217 - 32.

Vera, D. y Crossan, M.M. (2004): "Strategic leadership in organizational learning”, Academy of Management Review, 29, $222-240$.

Vieira, V.A. (2010): “Antecedents and Consequences of Market Orientation: a Brazilian Meta-Analysis and an International Mega-Analysis", Brazilian Administrative review, $7(1), 40-58$.

Vokurka, R.J. y Lummus, R. (2000): "The role of just in time in supply chain management", International Journal of Logistic Management, 11(1), 89 - 98.

Weick, K. (1987): “Organizational Culture as a Source of High Reliability", California Management Review, 29(2), 112.

Wernerfelt, B. (1984): “A resource-based view of the firm”, Strategic Management Journal, $5(2), 171-180$.

Wu, F., Sinkovics, R., Cavusgil, S. y Roath, A. (2007): "Overcoming Expert Manufacturers'Dilema in International Expansion", Journal of International Business Studies, 38 (2), 283 - 302.

Zahra, S. y George, G. (2002): "Absorptive capacity: a review reconceptualization and extension", Academy of Management Review, 27(2), 185 - 203.

Zysman, J. (1994): "How institutions create historically-rooted trajectories of growth", Industrial and Corporate Change, 3, $243-283$. 